\title{
Statistical uncertainty in the dark-field and transmission signal of grating interferometry
}

\author{
R. P. Harti, ${ }^{1,2, a)}$ M. Strobl, ${ }^{1,3,4}$ M. Morgano, ${ }^{1,3}$ J. Valsecchi, ${ }^{1,2}$ and C. Grünzweig ${ }^{1}$ \\ ${ }^{1}$ Laboratory for Neutron Scattering and Imaging, Paul Scherrer Institut, 5232 Villigen, Switzerland \\ ${ }^{2}$ Department of Quantum Matter Physics (DQMP), University of Geneva, 1205 Geneva, Switzerland \\ ${ }^{3}$ European Spallation Source ERIC, 22592 Lund, Sweden \\ ${ }^{4}$ Niels Bohr Institute, University of Copenhagen, 1017 Copenhagen K, Denmark
}

(Received 23 June 2017; accepted 10 October 2017; published online 27 October 2017)

\begin{abstract}
We present a framework to estimate the fundamental statistical uncertainty of grating interferometer experiments based on a Monte-Carlo method. Using the framework, we are able to determine the uncertainty of individual measurements as well as suggesting experimental protocols that minimise the statistical uncertainty for given overall exposure times. The method presented here is valid for both X-rays and neutrons and can be generalised for any modulation measurement. Published by AIP Publishing. https://doi.org/10.1063/1.4991760
\end{abstract}

\section{INTRODUCTION}

Dark-field images (DFIs) obtained by grating interferometry with X-rays $(\mathrm{xGI})^{1}$ and neutrons $(\mathrm{nGI})^{2,3}$ enable the extraction of spatial correlation functions in each pixel that yield information on structures well below the spatial resolution of the imaging system when altering the probed autocorrelation length of the setup. ${ }^{4-7}$ This paves the way for quantifying the retrieved changes of the DFI signal with varying autocorrelation length and allows the interpretation as the local autocorrelation function of the system in each pixel. ${ }^{8}$ In order to understand the accuracy of the data, the intrinsic uncertainty of the transmission image (TI) and the dark-field signal in grating interferometry has to be evaluated to make interpretations reliable. Previous studies on the errors in grating interferometry and especially of the dark-field signal investigated the noise level but did not consider the fundamental statistical limitations of measurements. ${ }^{9,10}$

Furthermore the exposure time of grating interferometry experiments is typically orders of magnitude longer than comparable transmission studies. Especially in nGI, the ratio of available time to required time is critical. Thus the experimental protocol should be optimised towards the lowest possible uncertainty in the data for a given exposure time.

The uncertainty evaluation in grating interferometer experiments needs to account for additional steps compared to conventional transmission imaging where the relation of measured values and the result is rather direct. Grating interferometry data reduction contains an extra step in which the pixel values from a stepping procedure of a grating are used to fit a sinusoidal function. ${ }^{11}$ The resulting fit contains the values of the TI as well as DFI.

In this work, we identify the most important contributors to the uncertainty in the DFI and TI in grating interferometry and suggest experimental protocols to minimise the uncertainty of the measurement. We consider fitting a

a)Electronic mail: Ralph.Harti@psi.ch. sinusoidal function on noisy simulated data points and thus account for all steps in a grating interferometer experiment. We furthermore present a way of simulating the expected fundamental uncertainty in the measurement and show how this can be used to assign an error bar to the retrieved values. This way we show how the reliability of the data can be judged and give a statistical backbone to quantitative interpretations.

While the statistical considerations of this work hold for both X-rays and neutrons, they are most noticeably for X-ray laboratory sources and neutrons as synchrotron experiments are typically conducted with much higher flux. For this work, we will focus on values that are typical for nGI. The reason for that is that neutron imaging experiments are likely to be dominated by statistical effects due to the limited flux of the available sources as well as the limited time available for experiments.

\section{MODEL AND VIRTUAL EXPERIMENT}

Our evaluation of the uncertainty in nGI follows the assumption of a Poisson-like behaviour of neutron statistics. This has been validated for neutron imaging previously ${ }^{12}$ and closely represents reality. We analyse the uncertainty that results purely from neutron statistics and do not consider uncertainties introduced by the detector, as these vary with each individual setup and have the potential to be minimised in future, while neutron statistics limitations are fundamental and cannot be circumvented. Therefore we do also not consider motor positioning problems, during G0 stepping, or detector efficiency as these are specific to experiments and are not a fundamental factor.

Accounting for those considerations, we designed a virtual experiment that allows us to freely vary the input parameters neutron count, number of grating phase steps, DFI value, TI value, and visibility. These parameters are the contributors to the fundamental statistical uncertainty in neutron grating interferometry. The nature of the statistical uncertainty does not allow any improvement on it and will 
always be present independent of the exact experimental conditions.

The algorithm making up the virtual experiment follows six steps.

Step one is the definition of a perfectly sinusoidal function with the statistics and behaviour of the previously defined input parameters. We assign a random phase to the sinusoidal to compensate for possible phase effects of fitting at a later stage which is also the reason why we do not analyse the uncertainty of the differential phase contrast. The perfect sinusoidals are defined for the open beam (OB) without the sample and the projection (proj) with the sample leading to a set of two equations,

$$
\begin{gathered}
y_{O B}\left(x_{g}\right)=a \cdot \sin \left(x_{g}-\phi_{r}\right)+N, \\
y_{\text {proj }}\left(x_{g}\right)=(a \cdot D F I) \cdot \sin \left(x_{g}-\phi_{r}\right)+(N \cdot T I) .
\end{gathered}
$$

$N$ is the average neutron count per pixel in each individual G0 phase step $x_{g}$ and $a$ is the amplitude which depends both on $N$ and the visibility. $\phi_{r}$ is the phase of the function which is kept random in order to mitigate phase effects. The modulation function for the sample $y_{p r o j}\left(x_{g}\right)$ is defined similar to $y_{O B}\left(x_{g}\right)$ with the additional contributions of the DFI and TI values. Figure 1 shows the resulting functions for both open beam and projection by plotting the neutron count over the number of phase steps.

Step two of the algorithm adds noise to the neutron count $N$ of the perfect sinusoidal functions of $y_{O B}\left(x_{g}\right)$ and $y_{p r o j}\left(x_{g}\right)$. This is achieved by adding a Poisson-like noise to each individual point within the phase step. This results in a deviation from the previously defined perfect function and is also plotted in Fig. 1 indicated by "Open Beam + noise" and "Projection + noise."

The noisy functions are then used in step three for nonlinear least square fitting in order to fit a sinusoidal function with fitting parameters $a, \phi_{r}$, and $N$ from Eqs. (1) and (2) to the noisy points. This results in amplitude and mean value of the fitted curve for both OB and proj. Due to the previously induced noise, the fitted parameters deviate from the initially perfectly defined function.

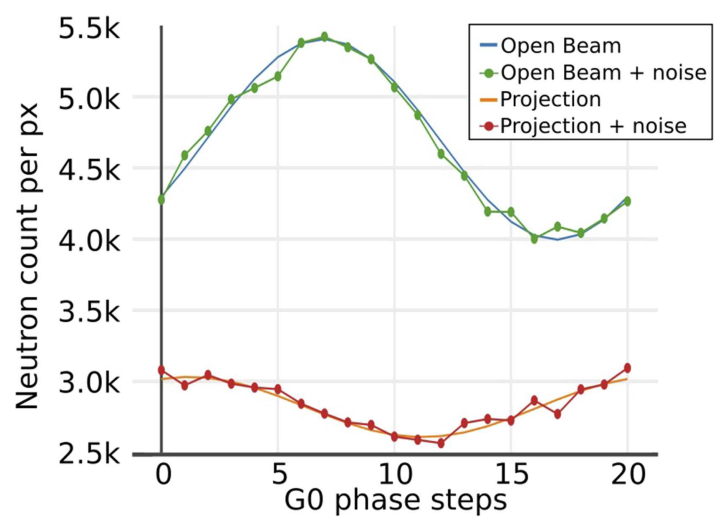

FIG. 1. Grating interferometry stepping functions indicating neutron count in each pixel with an average of 4700 neutrons for the open beam. Blue and orange show perfect sinusoidals. The projection (orange) has a lower mean value as well as a lower amplitude to represent TI and DFI values of 0.6 and 0.5 , respectively. The green and red plots have added Poisson noise that represents real statistics.
In step four, we use a Monte-Carlo technique to evaluate the confidence interval for that deviation. The confidence interval is determined by a Monte-Carlo approach with $n$ random and phase independent sinusoidals for OB and proj. That way we repeat steps one to three $\mathrm{n}$ times while defining the random phase and noise new for every iteration. We call $\mathrm{n}$ the sampling size which will be kept at 2500 unless specifically mentioned. We chose 2500 to fulfill the requirements for sample size of an infinite population ${ }^{13}$ following equation:

$$
n=\frac{z^{2} \cdot \sigma^{2}}{d^{2}}
$$

with $z$ being the critical value, $\sigma^{2}$ being the variance of the distribution, and $d$ being the margin of error. We assigned $z=1.96$ for a confidence level of $95 \%$ and $d=1$ to keep the margin of error below one neutron count. These considerations lead to a sample size of 1713 which we exceed by using 2500 . The margin of error for a sample size of 2500 is 0.83 and is thus conservatively kept below one neutron count. In order to evaluate the variance of the distribution, we ran the virtual experiment with an increased $n$ of 100000 and with the settings used for the example in Sec. III. This ensures an estimate of the variance that is above the achievable precision during experiments with $n=2500$. Furthermore we used the variance of the fitting parameter $a$ as we will focus on the error of the amplitude dependent DFI in Sec. III. The variance $\sigma^{2}$ was thus determined to be 446.72 for the specified settings. Even though the variance changes for each set of parameters for the virtual experiment, the exemplary calculation gives an indication of the margin of error that we can expect with a sample size of 2500. The Monte-Carlo approach with $\mathrm{n}=2500$ results in 2500 fitted mean values for the $\mathrm{OB}$ and the proj as well as 2500 fitted amplitudes for $\mathrm{OB}$ and proj. Figure 2 shows exemplary the histograms of mean and amplitude for a set of OB functions. The distribution of mean values is characterised by a standard deviation of 14.8 and the standard deviation of the amplitude by 21.3 . Since the DFI in grating interferometry is a comparison of amplitude between $\mathrm{OB}$ and proj, including transmission normalisation, and the TI is a comparison of mean values of OB and proj, we already expect an overall greater uncertainty in DFIs compared to TIs.

The value distributions in Fig. 2 together with their equivalents for the proj enable us to determine the confidence interval for $95 \%$ of the values. We define the confidence interval with an upper bound $\left(c_{u}\right)$ of

$$
c_{u}=m+h,
$$

and a lower bound $\left(c_{l}\right)$,

$$
c_{l}=m-h,
$$

with $m$ being the mean of the distribution. $\mathrm{h}$ is defined by

$$
h=s \cdot t(95 \%) \text {. }
$$

$\mathrm{s}$ is the standard error of the mean and $\mathrm{t}$ is the $\mathrm{t}$-score of the distribution for $95 \%$ confidence.

In step five, we get the standard deviations (std) for mean and amplitude of $\mathrm{OB}$ and proj from the determined confidence intervals by using

$$
s t d=\sqrt{n} \cdot\left(c_{u}-c_{l}\right) / 3.92 .
$$



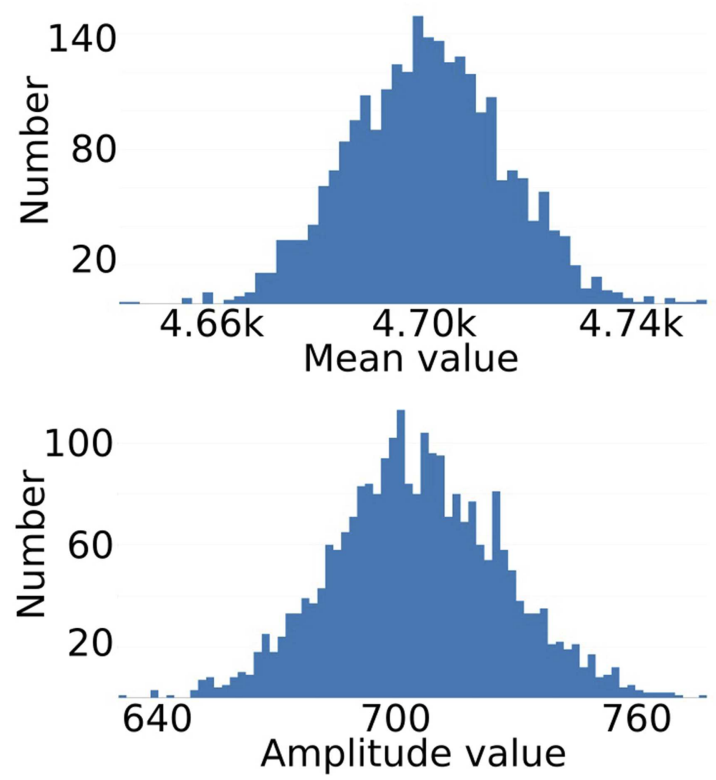

FIG. 2. Histograms of amplitude and mean value of 2500 simulated open beam functions showing the distribution of values extracted during the MonteCarlo simulation of noisy functions. The mean value distribution is characterised by a standard deviation of 14.8 and the amplitude value distribution by a standard deviation of 21.3 .

The factor 3.92 is a consequence of the width of a $95 \%$ confidence interval which is 3.92 standard errors of the distribution.

In the sixth and final step, we propagate the standard deviations of mean and amplitude for OB and proj through the equations that define transmission signal (TI) and the dark-field signal (DFI),

$$
\begin{gathered}
T I=\frac{a_{0}^{\text {proj }}}{a_{0}^{O B}}, \\
D F I=\frac{a_{1}^{p r o j} a_{0}^{O B}}{a_{0}^{p r o j} a_{1}^{O B} .}
\end{gathered}
$$

The subscript 0 for the values of $a$ indicates the mean value and the subscript 1 indicates the amplitude. The superscript proj stands for projection and $O B$ stands for the open beam. Error propagation through these equations results in the standard deviation for the TI $\left(T I_{e r r}\right)$ and for the DFI $\left(D F I_{e r r}\right)$ defined by

$$
\begin{gathered}
T I_{\text {err }}=\sqrt{\left(\frac{d T I}{d a_{0}^{p r o j}} \cdot \sigma a_{0}^{p r o j}\right)^{2}+\left(\frac{d T I}{d a_{0}^{O B}} \cdot \sigma a_{0}^{O B}\right)^{2},} \\
D F I_{\text {err }}=\sqrt{\frac{\left(\frac{d D F I}{d a_{1}^{p r o j}} \cdot \sigma a_{1}^{p r o j}\right)^{2}+\left(\frac{d D F I}{d a_{0}^{p r o j}} \cdot \sigma a_{0}^{p r o j}\right)^{2}}{d\left(\frac{d D F I}{d a_{0}^{O B}} \cdot \sigma a_{0}^{O B}\right)^{2}+\left(\frac{d T I}{d a_{1}^{O B}} \cdot \sigma a_{1}^{O B}\right)^{2}} .}
\end{gathered}
$$

These equations are the final step towards the determination of the uncertainty, and the presented uncertainties in Sec. III of this article are values resulting from these equation.

\section{RESULTS}

\section{A. TI and DFI uncertainty for a fixed set of parameters}

We use the determination of the statistical uncertainty as described above to create a model that evaluates the uncertainty in the DFI and TI as a consequence of input parameters. The input parameter space that impacts the statistical uncertainty is neutron count, DFI value, TI value, number of G0 steps, and visibility. The consequence is that for realistic values such as

- Neutron count per pixel: 4700

- DFI value: 0.5

- TI value: 0.6

- Number of G0 steps: 21

- Visibility: $15 \%$,

we are able to determine the statistical uncertainty and end up with a standard deviation for the DFI of 0.042 , accounting for $8.4 \%$ of the DFI signal. The standard deviation for the TI is one order of magnitude smaller and is determined to be 0.0031 or $0.52 \%$ of the signal.

\section{B. Uncertainty evolution with parameter space variation}

The model gives us the opportunity to study the impact of parameter space variations on the resulting uncertainty within the DFI. Here we will focus on the study of the uncertainty in DFIs due to its specificity for grating interferometry. A similar discussion for the TI signal can be found in the supplementary material. Figure 3 shows the uncertainty evolution for a variation of the parameter space defined above. We plot the change in the parameter as a function of the percentage of uncertainty in the DFI, which makes the uncertainty comparable for different sample conditions which we evaluate by plotting the uncertainty evolution for different TIs and DFIs.

While all parameters exhibit a similar behaviour, we can classify the effect on the uncertainty in two categories. Category one are the parameters that impact the uncertainty following a $\frac{1}{\sqrt{x}}$ law with $\mathrm{x}$ being the parameter in question. Category one is made up of parameters that change the total flux received by the experiment, namely, number of phase steps, neutron count, and TI value. These parameters increase the total amount of neutrons seen by the experiment and thus follow a Poisson behaviour. This behaviour is shown in Figs. 3(a) and 3(b) as the evolution of uncertainty versus neutron count and uncertainty versus phase steps.

Category two is made up of parameters that do not impact the total received flux but the measured amplitude. These are the measured DFI values itself as well as the visibility. These parameters most strongly influence the uncertainty in the DFI and follow a $\frac{1}{x}$ law with $\mathrm{x}$ being one of the parameters that influence the amplitude. The behaviour for DFI and visibility is shown in Figs. 3(c) and 3(d).

The categorical parameter classification suggests that the uncertainty is independent of the number of G0 phase steps used to generate the image, assuming that the overall flux is preserved and a minimum of three phase steps are recorded. 
a)
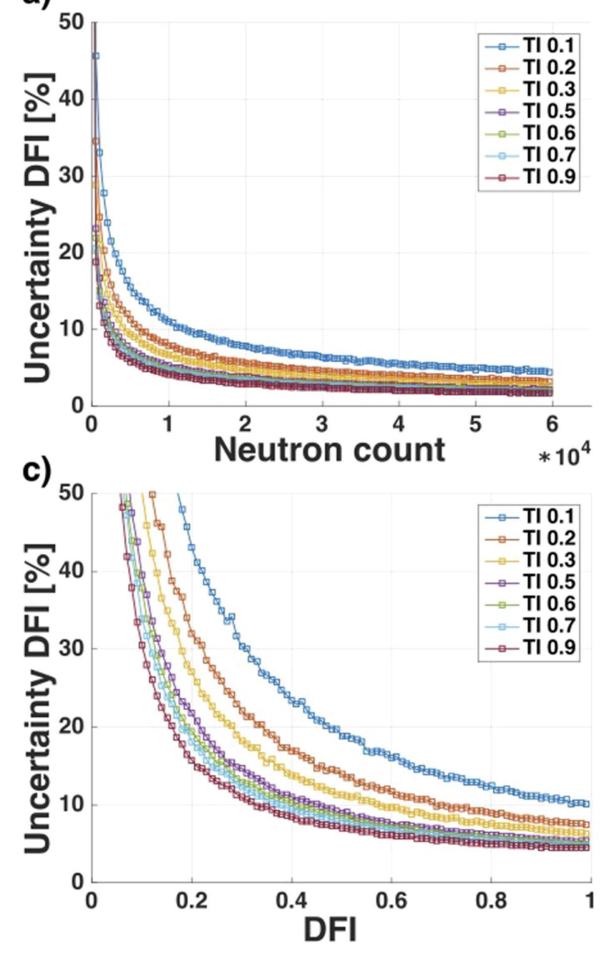

b)

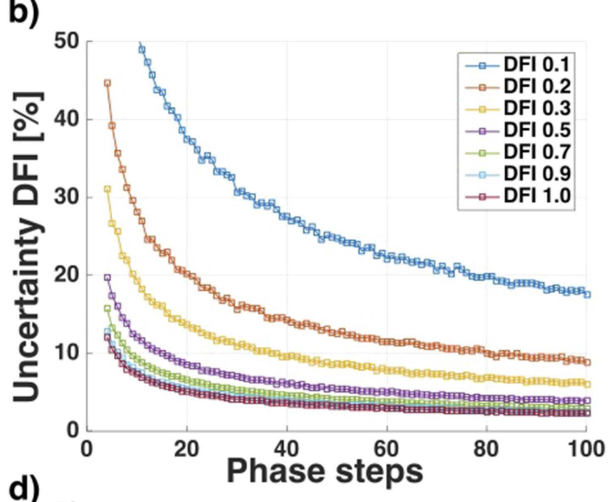

d)

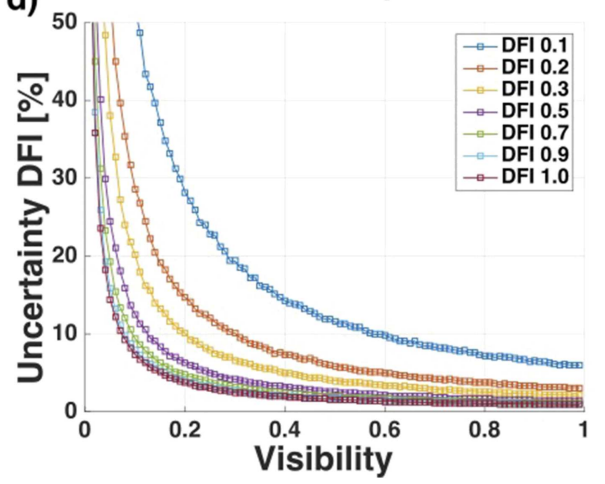

FIG. 3. DFI uncertainty behaviour with parameter variation. (a) shows the uncertainty evolution with increasing neutron count per pixel. For comparison, we show the behaviour for different TI values. (b) illustrates the uncertainty with increasing number of G0 phase steps and various DFI values. (c) shows the uncertainty as a function of DFI with additional variation of the TI value and (d) illustrates the impact of visibility for varying DFI values.

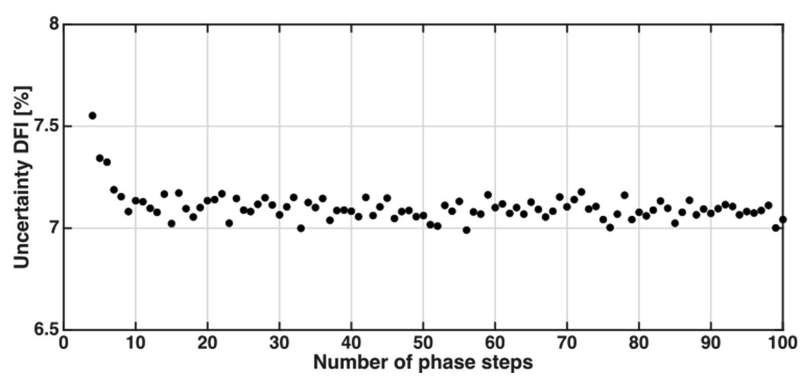

FIG. 4. DFI uncertainty behaviour with increasing number of phase steps while keeping the total neutron count constant. The presented values are determined with an overall neutron count of 160000 neutrons at a visibility of 0.15 and TI as well as DFI values of 0.5 .

Below three steps, the system is under determined and cannot be solved. However, as shown in Fig. 4, this behaviour does not hold for small numbers of steps. Up to 10 steps, the uncertainty in the DFI changes with the number of steps, despite using the same overall flux. These are results of the ambiguity in fitting few points with statistical noise. These results indicate that the uncertainty in the DFI cannot be reduced by a larger number of phase steps as long as the minimum number of 10 steps is reached. The uncertainty determination in Fig. 4 was done using $n=10000$ to reduce the margin of error and make more subtle changes visible.

\section{CONCLUSION AND DISCUSSION}

We presented a study on the fundamental, statistical uncertainty that contributes to uncertainties in the retrieval of darkfield and transmission images in grating interferometry. While the results are universal for grating interferometry, they seem to be most relevant for nGI, as well as for grating interferometry with X-ray tube sources, due to the limited flux. The considerations we outlined in this work can furthermore be generalised to determine statistical errors in any modulation measurement technique, such as spin echo methods ${ }^{14,15}$ and far-field interferometry. ${ }^{16}$ All in all, we showed that the unavoidable statistical uncertainty is not negligible and has to be taken into account while designing experimental procedures.

We characterised the uncertainty behaviour in dependence of varying experimental parameters and were able to categorise them into parameters that impact the received neutron count, thus the uncertainty follows a $\frac{1}{\sqrt{x}}$ and parameters that impact the contrast/amplitude and vary the uncertainty more rapidly, following a $\frac{1}{x}$ law. Thus experimental setups as well as experimental protocols should be designed to optimise the $\frac{1}{x}$ behaviour.

In particular, we identified the visibility as the major contributor to the statistical uncertainty. In combination with the findings in a previous study, ${ }^{17}$ we can conclude that improvements of the geometry of $\mathrm{G} 2$ will significantly decrease the uncertainty in the DFI as well as TI. Thus signal quantification is currently limited by the quality of analyser gratings which is the major contributor to reduced visibility in nGI.

From an experimental procedure point of view, the uncertainty of the DFI is independent of the number of steps used for the phase scan. Above a threshold of 10 steps per period, the uncertainty does not decrease significantly anymore, given a constant exposure time. We suggest to use 10 phase steps over one full period for future grating interferometry experiments to obtain the smallest uncertainty while keeping potential uncertainties introduced by motor movements minimal. 


\section{SUPPLEMENTARY MATERIAL}

See supplementary material for a discussion of the uncertainty in the transmission signal as a function of neutron count, phase steps, DFI, and visibility.

\section{ACKNOWLEDGMENTS}

The authors thank Christian Rüegg for the encouragement to do this study. This work is funded by the Swiss National Science Foundation (SNSF) (No. 153304).

${ }^{1}$ F. Pfeiffer, M. Bech, O. Bunk, P. Kraft, E. F. Eikenberry, C. Brönnimann, C. Grünzweig, and C. David, "Hard-x-ray dark-field imaging using a grating interferometer," Nat. Mater. 7, 134-137 (2008).

${ }^{2}$ C. Grünzweig, C. David, O. Bunk, M. Dierolf, G. Frei, G. Kühne, J. Kohlbrecher, R. Schäfer, P. Lejcek, H. M. R. Rønnow, and F. Pfeiffer, "Neutron decoherence imaging for visualizing bulk magnetic domain structures," Phys. Rev. Lett. 101, 025504 (2008).

${ }^{3}$ C. Grünzweig, J. Kopecek, B. Betz, A. Kaestner, K. Jefimovs, J. Kohlbrecher, U. Gasser, O. Bunk, C. David, E. Lehmann, T. Donath, and F. Pfeiffer, "Quantification of the neutron dark-field imaging signal in grating interferometry,” Phys. Rev. B 88, 125104 (2013).

${ }^{4}$ M. Strobl, "General solution for quantitative dark-field contrast imaging with grating interferometers," Sci. Rep. 4, 7243 (2014).

${ }^{5}$ F. Prade, A. Yaroshenko, J. Herzen, and F. Pfeiffer, "Short-range order in mesoscale systems probed by $\mathrm{x}$-ray grating interferometry," Europhys. Lett. 112, 68002 (2015).

${ }^{6}$ S. Gkoumas, P. Villanueva-Perez, Z. Wang, L. Romano, M. Abis, M. Stampanoni, C. David, A. Momose, T. Weitkamp, F. Pfeiffer, W. Yashiro, F. Pfeiffer, G. Potdevin, F. Schaff, T. Thüring, T. H. Jensen, T. Michel, M. Stampanoni, N. Hauser, Z. Wang, S. Schleede, F. G. Meinel, K. Scherer, S. K. Lynch, M. Strobl, W. Cong, A. Ritter, S. Peter, Y. Sung, F. Zernike, J. A. Prins, R. J. Baxter, S. Gkoumas, J. S. Pedersen, D. J. Kinning, E. L. Thomas, T. Donath, F. Prade, T. Thuering, J. Segur, and H. Oberstar, "A generalized quantitative interpretation of dark-field contrast for highly concentrated microsphere suspensions," Sci. Rep. 6, 35259 (2016).
${ }^{7}$ R. P. Harti, M. Strobl, B. Betz, K. Jefimovs, M. Kagias, and C. Grünzweig, "Sub-pixel correlation length neutron imaging: Spatially resolved scattering information of microstructures on a macroscopic scale," Sci. Rep. 7, 44588 (2017).

${ }^{8}$ M. Strobl, B. Betz, R. P. Harti, A. Hilger, N. Kardjilov, I. Manke, and C. Gruenzweig, "Wavelength-dispersive dark-field contrast: Micrometre structure resolution in neutron imaging with gratings," J. Appl. Crystallogr. 49, 569 (2016).

${ }^{9}$ M. Chabior, T. Donath, C. David, M. Schuster, C. Schroer, and F. Pfeiffer, "Signal-to-noise ratio in $\mathrm{x}$ ray dark-field imaging using a grating interferometer," J. Appl. Phys. 110, 053105 (2011).

${ }^{10}$ F. Schwab, S. Schleede, D. Hahn, M. Bech, J. Herzen, S. Auweter, F. Bamberg, K. Achterhold, A. Yildirim, A. Bohla, O. Eickelberg, R. Loewen, M. Gifford, R. Ruth, M. Reiser, K. Nikolaou, F. Pfeiffer, and F. Meinel, "Comparison of contrast-to-noise ratios of transmission and darkfield signal in grating-based $\mathrm{x}$-ray imaging for healthy murine lung tissue," Z. Med. Phys. 23, 236-242 (2013).

${ }^{11}$ S. Marathe, L. Assoufid, X. Xiao, K. Ham, W. W. Johnson, and L. G. Butler, "Improved algorithm for processing grating-based phase contrast interferometry image sets," Rev. Sci. Instrum. 85, 013704 (2014).

${ }^{12}$ F. Piegsa, A. Kaestner, A. Antognini, A. Eggenberger, K. Kirch, and G. Wichmann, "Statistical uncertainty in quantitative neutron radiography," Eur. Phys. J. Appl. Phys. 78, 10702 (2017).

${ }^{13}$ S. K. Thompson, "Sample size for estimating a population mean," in Sampling, 3rd ed., Wiley Series in Probability and Statistics (Wiley, 2012), Chap. 4.1, p. 54.

${ }^{14}$ M. Rekveldt, "Novel SANS instrument using neutron spin echo," Nucl. Instrum. Methods Phys. Res., Sect. B 114, 366-370 (1996).

${ }^{15}$ M. Strobl, M. Sales, J. Plomp, W. G. Bouwman, A. S. Tremsin, A. Kaestner, C. Pappas, and K. Habicht, "Quantitative neutron dark-field imaging through spin-echo interferometry," Sci. Rep. 5, 16576 (2015).

${ }^{16}$ D. A. Pushin, D. Sarenac, D. S. Hussey, H. Miao, M. Arif, D. G. Cory, M. G. Huber, D. L. Jacobson, J. M. LaManna, J. D. Parker, T. Shinohara, W. Ueno, and H. Wen, "Far-field interference of a neutron white beam and the applications to noninvasive phase-contrast imaging," Phys. Rev. A 95, 043637 (2017).

${ }^{17}$ R. P. Harti, C. Kottler, J. Valsecchi, K. Jefimovs, M. Kagias, M. Strobl, and C. Grünzweig, "Visibility simulation of realistic grating interferometers including grating geometries and energy spectra," Opt. Express 25, 1019 (2017). 